\title{
Early Switching Strategies in Antidepressant Non-Responders: Current Evidence and Future Research Directions
}

\author{
Paul A. Kudlow $\cdot$ Roger S. McIntyre • \\ Raymond W. Lam
}

Published online: 16 May 2014

(C) Springer International Publishing Switzerland 2014

\begin{abstract}
Studies have found that up to two-thirds of patients with major depressive disorder (MDD) do not fully respond to the first antidepressant. While switching antidepressants is a common strategy for antidepressant nonresponders, there is still a lack of consensus about the optimal timing of a switch. Many clinicians wait for 6-12 weeks before considering a switch. The objectives of this paper are to (1) review the evidence for positive and negative predictive value (NPV) of early improvement at 2-4 weeks to predict final antidepressant response; (2) review randomized controlled trials (RCTs) that examine early switching strategies; and (3) provide future research directions and clinical recommendations for timing of antidepressant switching. We conducted a literature search for English-language studies via PubMed and Google Scholar, from 1984 to May 2013, with the following terms: 'antidepressants', 'MDD', 'time course', 'trajectory', 'early response', 'onset', 'delayed response', 'early improvement', 'predictors', 'switch', 'combination therapy', and 'augmentation'. Replicated evidence indicates
\end{abstract}

P. A. Kudlow

Department of Psychiatry, University of Toronto, Toronto, ON, Canada

R. S. McIntyre

University of Toronto, Toronto, ON, Canada

R. S. McIntyre

Head, Mood Disorders Psychopharmacology Unit, University

Health Network, Toronto, ON, Canada

R. W. Lam ( $ه)$

Department of Psychiatry, University of British Columbia

(UBC) and Mood Disorders Centre of Excellence, UBC

Hospital, 2255 Wesbrook Mall, Vancouver, BC V6T 2A1,

Canada

e-mail: r.lam@ubc.ca that lack of early improvement (e.g. $<20 \%$ reduction in a depression scale score) at $2-4$ weeks can be an accurate predictor to identify eventual non-responders. The NPVs suggest that only about one in five patients with lack of improvement at 4 weeks will have a response by 8 weeks. Three RCTs examined early switch strategies, but results are inconsistent and comparisons limited by methodological differences. Future studies should incorporate a standard consensus definition of early improvement, discern whether the effect of early switching is specific to certain types of antidepressants, and determine whether early switch is superior to other strategies such as augmentation or combination. Notwithstanding these limitations, there is reasonable evidence to recommend earlier assessment for improvement. If there is no indication of early improvement at 2-4 weeks after starting an antidepressant, and taking into account other patient and clinical factors, a change in management can be considered.

\section{Key Points}

Lack of early improvement, defined by $<20 \%$ reduction in a depression rating scale at 2-4 weeks, may be predictive of eventual clinical non-response to an antidepressant.

In the absence of early improvement, and taking into account side effects and other clinical factors, a change in management (increase dose, switch to another antidepressant, augment with another medication, add psychotherapy) can be considered.

There is still limited quality evidence comparing early switch strategies to continuing an antidepressant. 


\section{Introduction}

According to clinical guidelines, antidepressant medications are considered among the first-line treatments for major depressive disorder (MDD) [1, 2]. While the evidence has shown that antidepressants are an effective treatment for moderate to severe MDD [3], naturalistic studies indicate that up to two-thirds of patients do not respond to the first antidepressant medication [4], with the chance of response decreasing and recurrence significantly increasing with subsequent treatment failures [5]. Most clinical guidelines recommend switching to another antidepressant as a first-line strategy for non-responders, although augmentation and combination strategies also have evidence to support their use. A salient clinical question remains: for patients who fail to respond to their initial antidepressant, what is the optimum time for switching? If patients are switched too early, they may needlessly abandon an effective medication; on the other hand, staying on an ineffective treatment will affect adherence and prolong suffering and impairment.

To date, a consensus still does not exist as to (a) when patients begin to improve after starting an antidepressant; and (b) how long patients should stay on an antidepressant before it is deemed ineffective. Evidence derived from older antidepressant studies suggested a delayed onset of therapeutic response [6-9], leading to the conventional belief that patients need to wait at least 8 weeks before concluding whether or not they are responding to an antidepressant [10]. However, recent reviews [10, 11] have summarized the many subsequent studies that showed an early onset (within 1-2 weeks) of antidepressant effects in ultimate responders, thereby contradicting the delayed-onset hypothesis and suggesting that responders can be identified earlier in the course of treatment.

Many studies have now examined whether early improvement, or lack of improvement, in depression symptoms can predict final clinical response. Most studies have defined 'early improvement' as $\geq 20 \%$ reduction from baseline on a depression rating scale (e.g. Hamilton Rating Scale for Depression [HAM-D] [12], Montgomery Asberg Depression Rating Scale [MADRS]) [13], while 'clinical response' is typically defined as $\geq 50 \%$ reduction from baseline. An early study of fluoxetine response found that early improvement at 2 weeks could predict $75 \%$ of eventual responders [14]. Furthermore, other studies have suggested that lack of early improvement at 2 weeks may serve to reliably identify a subpopulation of non-responders [11]. Having this non-responder subpopulation wait 8-12 weeks before switching to another antidepressant may not be justified.
If early improvement can predict final response, how might this guide clinical decisions? One important clinical metric is the positive predictive value (PPV), which is the probability that a positive test (in this case, early improvement) actually results in a positive outcome (in this case, response at the end of the study). For clinicians, a more clinically relevant metric is the negative predictive value (NPV), which is the probability that a negative test (lack of early improvement) leads to a negative outcome (lack of response). A high NPV suggests that a response is unlikely with continued use of the antidepressant, and a change in management, such as switching to another medication, should be considered.

The objectives of this paper are to (1) review the evidence for predictive value of early improvement or lack of improvement for end-of-study response, focusing on studies examining PPV and NPV for current first-line antidepressants; (2) review controlled studies that examine the timing of early switching strategies; and (3) provide future research directions and clinical recommendations for timing of antidepressant switching.

A non-systematic review of the literature was conducted using PubMed and Google Scholar. The search included all English-language articles published between 1984 and May 2013. The following search terms were used: 'antidepressants', 'major depressive disorder', 'time course', 'trajectory', 'early response', 'onset', 'delayed response', 'early improvement', 'predictors', 'switch', 'combination therapy', and 'augmentation'. The terms were cross-referenced with each other to achieve the most comprehensive and relevant results. Bibliographies were also reviewed for further citations. Articles selected for narrative review were based on adequacy of sample size, the use of standardized experimental procedures, validated assessment measures, and overall methodological quality. We focused our review on studies involving newer antidepressants (selective serotonin reuptake inhibitors [SSRIs], serotonin norepinephrine reuptake inhibitors [SNRIs], bupropion, mirtazapine and agomelatine) because they are most commonly used in initial treatment.

\section{Predictive Value of Early Improvement for Antidepressant Response}

Table 1 summarizes the identified studies using early improvement to predict antidepressant outcome in which the PPV (13 studies) and/or NPV (15 studies) were reported or could be calculated from the results. For tabulation of NPV and PPV, we included only data for clinical response and definitions of early improvement based on change in a depression symptom scale. We chose to focus 


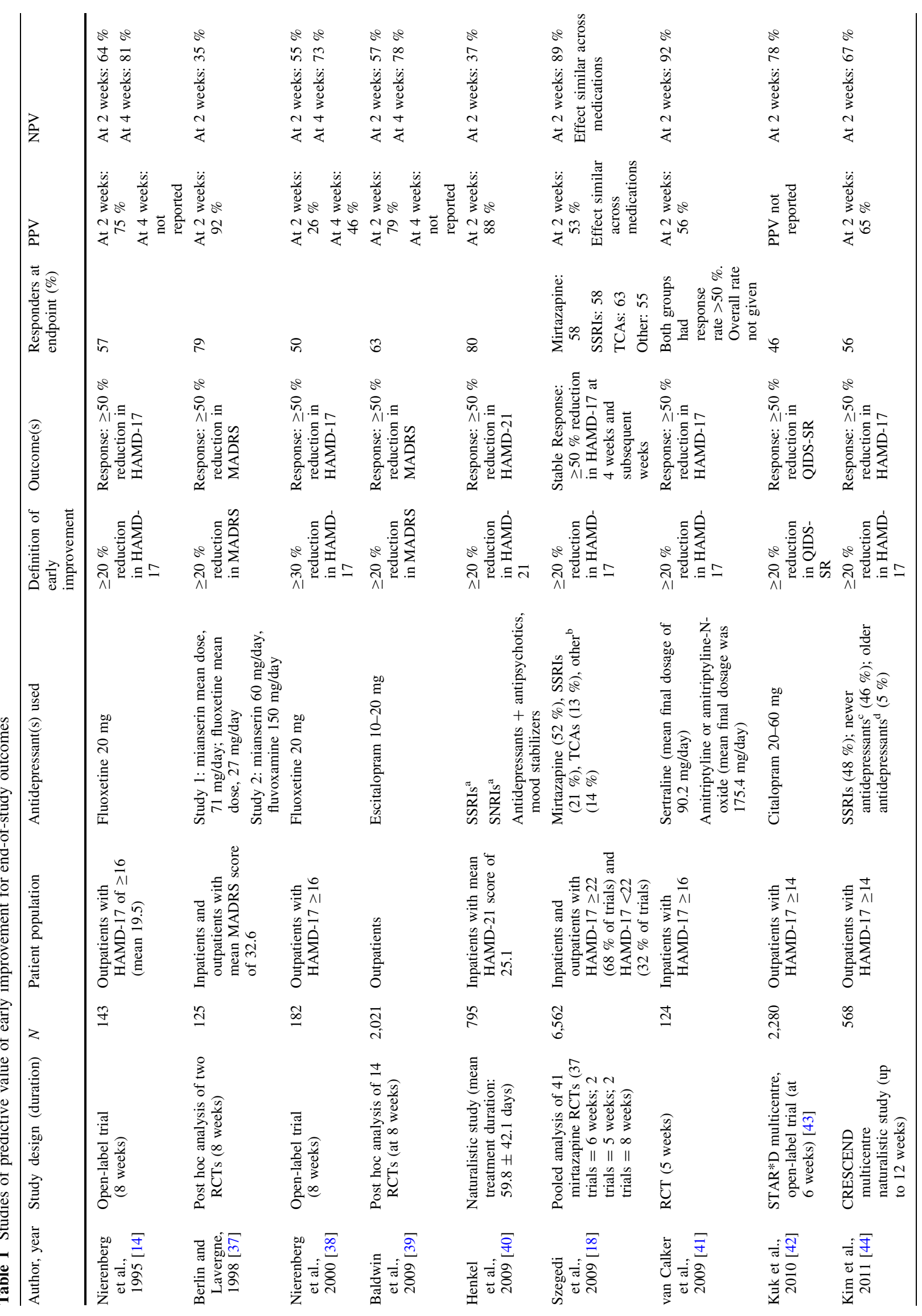




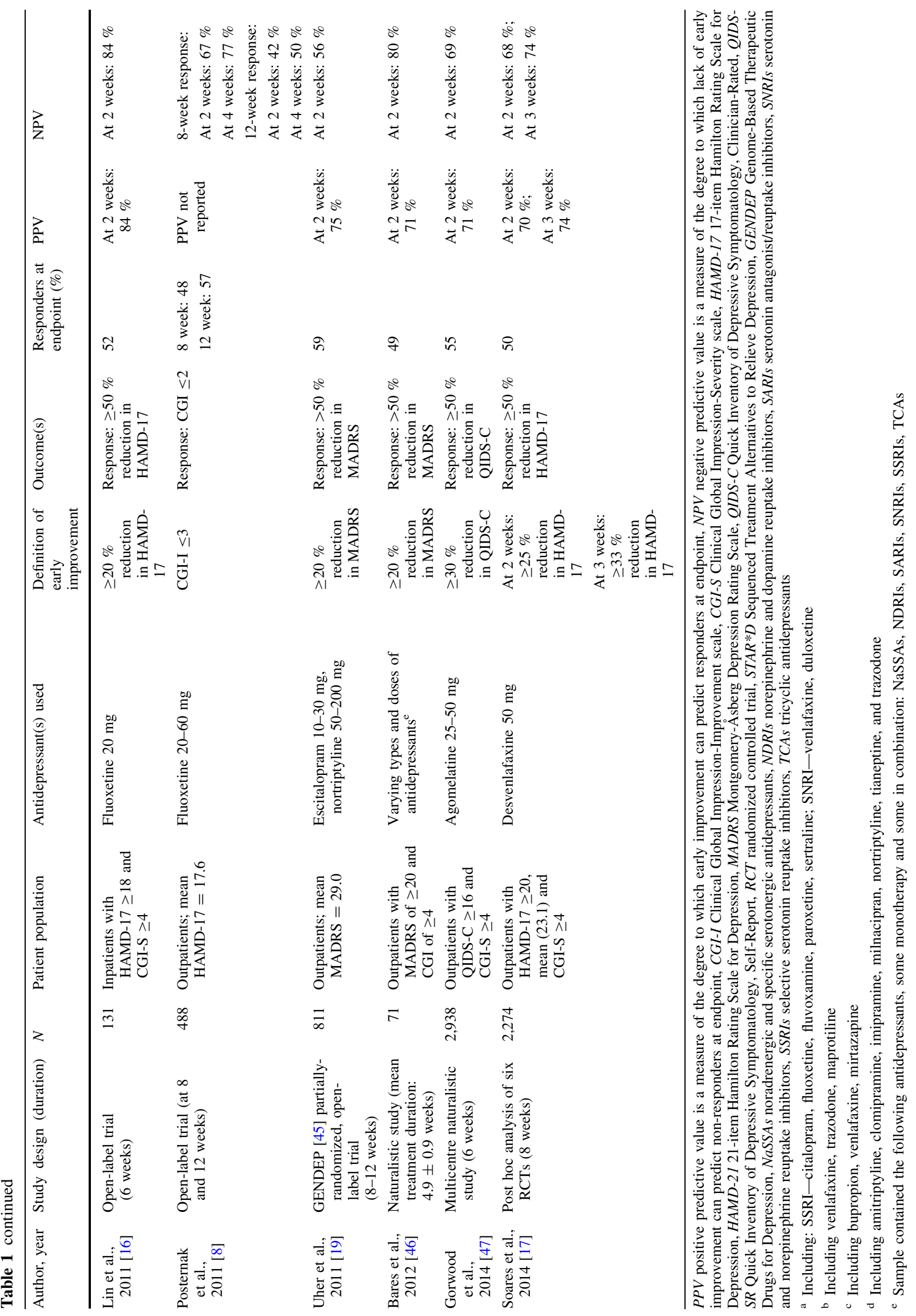


on clinical response instead of remission to maximize comparisons across studies and because of variations in the definition of remission. In addition, remission rates vary depending on study duration, making it difficult to compare 6- to 12-week studies. We excluded two studies because they only included remission data $[9,15]$.

Most of the identified studies were open-label or naturalistic trials ( $n=10$ studies) or pooled meta-analyses of randomized controlled trials (RCTs; $n=4$ studies) with sample sizes ranging from 71 to 6,562. The studies reported end-of-study response rates ranging from $46 \%$ to $80 \%$ after 6 weeks ( $n=6$ studies), 8 weeks $(n=7$ studies), and up to 12 weeks ( $n=3$ studies). Early improvement was usually defined as at least $20 \%$ reduction in HAM-D or MADRS ( $n=11$ studies). Two studies also examined optimum definitions of early improvement using receiver operating characteristic analysis [16, 17]. For early improvement at 2 weeks, PPVs were $26-84 \%$, while NPVs were 35-92\%. Five studies also included early improvement prediction at 3-4 weeks, with NPVs of 73-81\%. Moreover, the NPV was either higher or similar to the PPV in the majority of studies $(10 / 15,67 \%)$, suggesting that NPV is a more reliable predictor. An example is the largest study, a meta-analysis of individual patient data $(n=6,562)$ from 14 RCTs [18], using early improvement (at least $20 \%$ reduction in HAMD-17 at 2 weeks) as a predictor of stable response at 6 weeks. The PPV was relatively modest in that $53 \%$ of early improvers became stable responders. However, the NPV was high at $89 \%$, indicating that only $11 \%$ of patients without early improvement at 2 weeks became stable responders at 6 weeks.

In contrast, some studies have not found that early improvement predicted later response [8, 19]. For example, results of the partly randomized Genome-Based Therapeutic Drugs for Depression (GENDEP) study of escitalopram and nortriptyline $(n=811)$ determined that early improvement or lack of improvement at 2 or 3 weeks did not predict final response or remission at 12 weeks [19]. In the study, the timing of response was found to follow on a continuum; however, an accurate prediction of response only become clear following 8 weeks of treatment.

Despite these few discrepant studies, replicated evidence indicates that lack of early improvement at 2 weeks can be an accurate early predictor to identify eventual non-responders. The NPVs for 4 weeks are even higher than 2 weeks; only about one in five patients with lack of improvement at 4 weeks will have a response by 8 weeks. In the following section, we will examine the evidence for the optimal timing of treatment-switching strategies in this early non-improver MDD subpopulation.

\section{Controlled Studies of Early-Switch Strategies}

While the benefits of switching antidepressant medications after a failed trial have been investigated in many openlabel studies and RCTs [20-22], only three studies have investigated the timing of switch strategies for antidepressant non-responders (Table 2). Nakajima et al. [23] completed a small sample $(n=41)$, randomized, openlabel trial that showed benefits of an early-switch strategy versus maintaining the same antidepressant in individuals who did not show early improvement. Patients $(n=132)$ were initially treated with sertraline $50 \mathrm{mg}$; non-improvers ( $<20 \%$ reduction in MADRS) at 2 weeks were randomized to 6 weeks in one of two groups: (a) sertraline was continued and titrated at $50-100 \mathrm{mg}(n=21)$; (b) sertraline was switched to paroxetine $20-40 \mathrm{mg}(n=20)$. For the primary outcome, the early-switch group showed a higher rate of responders than the continuing titration group ( 75 vs. $19 \% ; p=0.002$ ). In secondary outcomes, the early-switch group was also superior in the rate of remitters (MADRS $\leq 10)$ [ $60 \%$ vs. $14 \% ; p=0.004]$ and change in MADRS scores $(19.0$ vs. $7.5 ; p<0.001)$. Of note is that these results may be limited by the differential dosing of the antidepressants, since maximal doses $(40 \mathrm{mg}$ ) of paroxetine were possible while submaximal doses of sertraline were used.

In contrast, Bose et al. [24] found support for increasing the dose of the index antidepressant compared with an early-switch strategy. Severely depressed (MADRS $\geq 30$ at baseline) patients with MDD $(n=571)$ received singleblind treatment with escitalopram $10 \mathrm{mg} / \mathrm{day}$; nonresponders $(<50 \%$ reduction in MADRS) at week 2 were randomized to 8 weeks of treatment with up-titration to escitalopram $20 \mathrm{mg}(n=229)$ or switch to duloxetine $60 \mathrm{mg}(n=245)$. At endpoint, there was no significant difference between conditions in the primary outcome, allcause premature study discontinuations, or in MADRS response rates. However, the escitalopram up-titration group had significantly greater reduction in MADRS total score, as well as significantly higher remission rates (MADRS $\leq 10$ ) than the duloxetine-switch group (54\% vs. $42 \%$, respectively; $p=0.013$ ).

The third study examined an early (4 weeks) versus a more conventional ( 8 weeks) switch strategy in patients who did not show early improvement [25]. Patients with MDD $(n=840)$ were initially treated with escitalopram $10 \mathrm{mg}$. Patients who did not achieve early improvement ( $\leq 30 \%$ reduction in HAM-D) at week 4 were randomized to switch to duloxetine (flexibly dosed at $60-120 \mathrm{mg} /$ day) [early switch, $n=282$ ] for 12 weeks, or continue on escitalopram (flexibly dosed at $10-20 \mathrm{mg} /$ day) with nonresponders $(<50 \%$ reduction in HAM-D) at week 8 , switching to flexibly-dosed duloxetine (conventional 


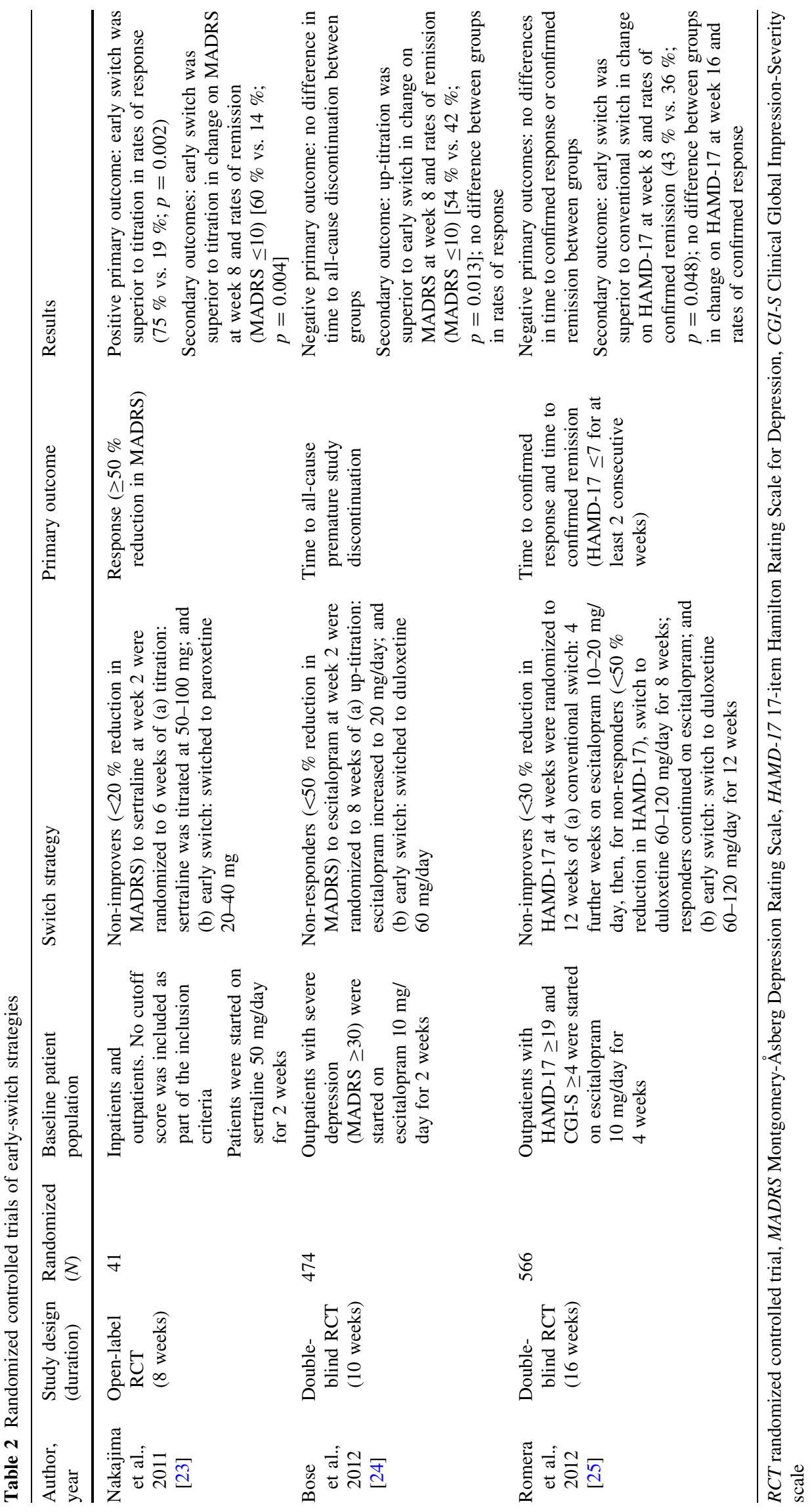


switch, $n=284$ ) for another 8 weeks. Hence, the conventional-switch group was composed of patients who responded to escitalopram and stayed on the medication for 16 weeks $(n=83)$, and those who were on escitalopram for 8 weeks and duloxetine for 8 weeks $(n=165)$. At the end of 16 weeks, there were no statistically significant differences between the two strategies in the primary outcomes, time to confirmed response ( $\geq 50 \%$ reduction in HAM-D for at least 2 consecutive weeks) or confirmed remission (HAM-D $\leq 7$ for at least 2 consecutive weeks). However, those subjects randomized to the early-switch strategy achieved significantly greater confirmed remission rates compared with the delayed switch group (43\% vs. $36 \%$, respectively; $p=0.048$ ). A pre-specified subgroup analysis examined patients with pain at baseline $(n=434)$ [26]. Those in the early-switching group $(n=138)$ were found to have significantly lower visual analogue scale mean pain levels for overall pain, headache, back pain, shoulder pain, interference with daily activities, and time being awake in pain compared with the delayed-switching group $(n=89)$. Moreover, time to achieving normal functioning (Sheehan Disability Scale total score $\leq 6$ [27]) was shorter in the early-switching group $(p=0.042)$ [26].

In summary, a small study found that an early switch (at 2 weeks) from sertraline to paroxetine led to superior outcomes compared with continuing on sertraline [23]. In contrast, both larger studies had negative results for primary outcomes for an early switch; only on analysis of secondary outcomes did some benefits of different strategies become apparent. However, one study found superior secondary outcomes for up-titration of escitalopram compared with early switch to duloxetine [24], while the other found superior outcomes for early switch (at 4 weeks) to duloxetine compared with a conventional switch (at 8 weeks) strategy [25].

\section{Limitations of the Evidence}

Unfortunately, it is difficult to reconcile the results of these studies because of differences in methodology, including the definitions of early improvement, the timing of switch decisions, the type of antidepressant switches, and the primary outcomes. Lack of early improvement, and the randomization to switch, was defined as $<20 \%$ reduction in MADRS at 2 weeks [23], $<50 \%$ reduction on the MADRS at 2 weeks [24], and $<30 \%$ improvement on the HAM-D at 4 weeks [25]. One study switched from one SSRI to another [23], while the other two involved SSRI to SNRI switches. The question of whether a switch within an antidepressant class is superior to switching to another class is still in doubt. A recent study [28] indicated that in patients unresponsive to SSRIs, changing to antidepressants with different mechanisms of action may be a more effective switching strategy. Generally, however, systematic reviews and large RCTs have either shown no differences $[20,21]$ in switching from one SSRI to another versus a different class, or small differences of questionable clinical significance [22].

\section{Future Research Directions}

Given the limitations and inconsistent findings of the few studies investigating early-switch strategies, further RCTs are needed to replicate and clarify these data and to discern whether any benefits of early switching are specific to within-class or between-class switches. Notwithstanding, there is still a lack of consensus as to whether early improvement with antidepressants is specific to certain mechanisms of action, or common across medications. Given the lack of consensus, additional RCTs that directly compare dissimilar antidepressants (e.g. SSRIs versus SNRIs versus other newer agents, etc.) are needed in order to determine whether class/mechanism of action affects onset of antidepressant effects. Future studies should incorporate a standard consensus definition of early improvement.

There are also no studies to date that have examined the timing of other commonly practiced treatment interventions such as augmentation with lithium or atypical antipsychotics, or combination antidepressant therapy. Rigorous RCTs are needed in order to determine the clinical benefits associated with the optimal timing of these treatment interventions compared with switch strategies.

While traditional clinical scales, such as the HAM-D and MADRS, may be sufficient for detection of overall symptom improvement, they may not be the optimal scales for detecting early improvement. Some symptom items (e.g. insomnia) are prone to improve or worsen early in treatment as a consequence of side effects of the antidepressant, and hence will confound the assessment of early improvement. There is also some evidence that certain depressive symptoms may improve earlier and to a greater extent than other core symptoms [11, 15, 29]. Further research into new clinical metrics, possessing greater item sensitivity and specificity for detection of these early effects of antidepressant therapy, is required. Self-rated scales (e.g. the Quick Inventory for Depressive Symptomatology, Self-Rated [QIDS-SR] [30]), which are more feasible to use in busy clinical practice settings, should also be examined in addition to clinician-rated scales. Additionally, studies have focused on prediction of eventual symptom-based definitions of clinical response or remission. Because there is increasing recognition that functional improvement and quality of life are more important than 
symptom improvement as a treatment outcome for patients with MDD [31, 32], future research should also examine early symptom improvement as a predictor of functional and quality-of-life outcomes.

A high priority for future research is to identify biomarkers (e.g. genetic, genomic and proteomic factors, neuroimaging, electroencephalography, polysomnography [33], etc.) of early improvement and to determine whether these can help predict response trajectories and identify those patients who would most benefit from early treatment switches. For example, a recent RCT provides evidence for using functional neuroimaging (i.e. changes in insula metabolism) as a 'treatment-specific biomarker' which can be used as an objective guide for whether to initiate pharmacotherapy or cognitive behavioral therapy in individuals with MDD [34]. Additional studies to elucidate other biomarkers of early improvement and prediction of treatment response are currently underway [35].

\section{Conclusions and Clinical Recommendations}

The findings from these various studies support the principles of measurement-based care, or the routine use of clinical scales, to properly assess for early improvement and response trajectories. Notwithstanding the limitations described, there is sufficient evidence to recommend earlier assessment for lack of improvement. If there is no indication of early improvement (e.g. $<20$ or $<30 \%$ reduction in depression rating scale scores) at 2-4 weeks after starting an antidepressant, a change in management can be considered. The decision for a change, or to continue 'watchful waiting', must also take into account other patient and clinical factors, including the severity of depression, need for rapid improvement (including risk for suicidality and severe functional impairment), psychosocial context and complicating stressors or life events, side effects experienced, physical or psychiatric comorbidities, and patient preference. A change in management could include increasing the dose of antidepressant, switching to another antidepressant, augmenting with another agent, or adding a non-medication treatment such as psychotherapy. If the decision is made to switch antidepressants, care should be taken to avoid discontinuation syndrome by tapering (reduction or increase of regimen dose/frequency over time) or cross-tapering antidepressants (one antidepressant is tapered down while the new antidepressant is tapered up over the same period of time) [36].

Results of future research may allow for a more personalized approach to the treatment of MDD as it may help to inform clinicians as to which individuals may benefit from early pharmacological treatment interventions (i.e. switch, combination, augmentation), non-pharmacological approaches, or those who would alternatively benefit from staying the course. Similarly, a validated biomarker to identify people who will not benefit from an ongoing antidepressant treatment at the earliest possible occasion could minimize unnecessary drug exposure and suffering.

Acknowledgements Paul A. Kudlow declares no conflicts of interest. Roger S. McIntyre has received research funding from AstraZeneca, GlaxoSmithKline, Merck, Servier, and Wyeth, and has served as a consultant to, and on the speaker's boards for, AstraZeneca, Eli Lilly, GlaxoSmithKline, Lundbeck, Organon, OrthoMcNeil-Janssen, Oryx, Pfizer, Prestwick, and Wyeth. Raymond W. Lam is on ad hoc speaker/advisory boards for, or has received research funds from, AstraZeneca, Biovail, Bristol-Myers Squibb, Canadian Institutes of Health Research, Canadian Network for Mood and Anxiety Treatments, Canadian Psychiatric Association Foundation, Eli Lilly, Litebook Company, Lundbeck, Lundbeck Institute, Mochida, Pfizer, Servier, St. Jude's Medical, Takeda, and UBC Institute of Mental Health/Coast Capital Savings. None of these interests had relevance to our review. No funding was obtained for the writing of this review.

\section{References}

1. Anderson IM, Ferrier IN, Baldwin RC, et al. Evidence-based guidelines for treating depressive disorders with antidepressants: a revision of the 2000 British Association for Psychopharmacology guidelines. J Psychopharmacol. 2008;22(4):343-96.

2. Davidson JR. Major depressive disorder treatment guidelines in America and Europe. J Clin Psychiatry. 2010;71(Suppl E1):e04.

3. Lam RW, Kennedy SH, Grigoriadis S, et al. Canadian Network for Mood and Anxiety Treatments (CANMAT) clinical guidelines for the management of major depressive disorder in adults. III: pharmacotherapy. J Affect Disord. 2009;117(Suppl 1):S26-43.

4. Trivedi MH, Rush AJ, Wisniewski SR, et al. Evaluation of outcomes with citalopram for depression using measurement-based care in STAR*D: implications for clinical practice. Am J Psychiatry. 2006;163(1):28-40.

5. Pae CU, Wang SM, Lee SY, et al. Early switch strategy in patients with major depressive disorder. Expert Rev Neurother. 2012;12(10):1185-8.

6. Quitkin FM, Rabkin JG, Ross D, et al. Duration of antidepressant drug treatment. What is an adequate trial? Arch Gen Psychiatry. 1984;41(3):238-45.

7. Quitkin FM, McGrath PJ, Stewart JW, et al. Chronological milestones to guide drug change. When should clinicians switch antidepressants? Arch Gen Psychiatry. 1996;53(9):785-92.

8. Posternak MA, Baer L, Nierenberg AA, et al. Response rates to fluoxetine in subjects who initially show no improvement. J Clin Psychiatry. 2011;72(7):949-54.

9. Quitkin FM, Petkova E, McGrath PJ, et al. When should a trial of fluoxetine for major depression be declared failed? Am J Psychiatry. 2003;160(4):734-40.

10. Lam RW. Onset, time course and trajectories of improvement with antidepressants. Eur Neuropsychopharmacol. 2012;22(Suppl 3):S492-8.

11. Kudlow PA, Cha DS, McIntyre RS. Predicting treatment response in major depressive disorder: the impact of early symptomatic improvement. Can J Psychiatry. 2012;57(12):782-8.

12. Hamilton M. A rating scale for depression. J Neurol Neurosurg Psychiatry. 1960;23:56-62.

13. Montgomery SA, Asberg M. A new depression scale designed to be sensitive to change. Br J Psychiatry. 1979;134:382-9. 
14. Nierenberg AA, McLean NE, Alpert JE, et al. Early nonresponse to fluoxetine as a predictor of poor 8-week outcome. Am J Psychiatry. 1995;152(10):1500-3.

15. Katz MM, Meyers AL, Prakash A, et al. Early symptom change prediction of remission in depression treatment. Psychopharmacol Bull. 2009;42(1):94-107.

16. Lin CH, Lane HY, Chen CC, et al. Early prediction of fluoxetine response for Han Chinese inpatients with major depressive disorder. J Clin Psychopharmacol. 2011;31(2):187-93.

17. Soares CN, Fayyad RS, Guico-Pabia CJ. Early improvement in depressive symptoms with desvenlafaxine $50 \mathrm{mg} / \mathrm{d}$ as a predictor of treatment success in patients with major depressive disorder. J Clin Psychopharmacol. 2014;34(1):57-65.

18. Szegedi A, Jansen WT, van Willigenburg AP, et al. Early improvement in the first 2 weeks as a predictor of treatment outcome in patients with major depressive disorder: a metaanalysis including 6562 patients. J Clin Psychiatry. 2009; 70(3):344-53.

19. Uher R, Mors O, Rietschel M, et al. Early and delayed onset of response to antidepressants in individual trajectories of change during treatment of major depression: a secondary analysis of data from the Genome-Based Therapeutic Drugs for Depression (GENDEP) study. J Clin Psychiatry. 2011;72(11):1478-84.

20. Rush AJ, Trivedi MH, Wisniewski SR, et al. Bupropion-SR, sertraline, or venlafaxine-XR after failure of SSRIs for depression. N Engl J Med. 2006;354(12):1231-42.

21. Ruhe HG, Huyser J, Swinkels JA, et al. Switching antidepressants after a first selective serotonin reuptake inhibitor in major depressive disorder: a systematic review. J Clin Psychiatry. 2006;67(12):1836-55.

22. Papakostas GI, Fava M, Thase ME. Treatment of SSRI-resistant depression: a meta-analysis comparing within- versus acrossclass switches. Biol Psychiatry. 2008;63(7):699-704.

23. Nakajima S, Uchida H, Suzuki T, et al. Is switching antidepressants following early nonresponse more beneficial in acute-phase treatment of depression?: a randomized open-label trial. Prog Neuropsychopharmacol Biol Psychiatry. 2011;35(8):1983-9.

24. Bose A, Tsai J, Li D. Early non-response in patients with severe depression: escitalopram up-titration versus switch to duloxetine. Clin Drug Investig. 2012;32(6):373-85.

25. Romera I, Perez V, Menchon JM, et al. Early switch strategy in patients with major depressive disorder: a double-blind, randomized study. J Clin Psychopharmacol. 2012;32(4):479-86.

26. Romera I, Perez V, Menchon JM, et al. Early vs. conventional switching of antidepressants in patients with MDD and moderate to severe pain: a double-blind randomized study. J Affect Disord. 2012;143(1-3):47-55.

27. Sheehan KH, Sheehan DV. Assessing treatment effects in clinical trials with the discan metric of the Sheehan Disability Scale. Int Clin Psychopharmacol. 2008;23(2):70-83.

28. Rosso G, Rigardetto S, Bogetto F, et al. A randomized, singleblind, comparison of duloxetine with bupropion in the treatment of SSRI-resistant major depression. J Affect Disord. 2012; 136(1-2):172-6.

29. Harmer CJ, Goodwin GM, Cowen PJ. Why do antidepressants take so long to work? A cognitive neuropsychological model of antidepressant drug action. Br J Psychiatry. 2009;195(2):102-8.

30. Rush AJ, Trivedi MH, Ibrahim HM, et al. The 16-item Quick Inventory of Depressive Symptomatology (QIDS), clinician rating (QIDS-C), and self-report (QIDS-SR): a psychometric evaluation in patients with chronic major depression. Biol Psychiatry. 2003;54(5):573-83.

31. Lam RW, Filteau MJ, Milev R. Clinical effectiveness: the importance of psychosocial functioning outcomes. J Affect Disord. 2011;132(Suppl 1):S9-13.
32. Ishak WW, Balayan $\mathrm{K}$, Bresee $\mathrm{C}$, et al. A descriptive analysis of quality of life using patient-reported measures in major depressive disorder in a naturalistic outpatient setting. Qual Life Res. 2013;22(3):585-96.

33. Kudlow PA, Cha DS, Lam RW, et al. Sleep architecture variation: a mediator of metabolic disturbance in individuals with major depressive disorder. Sleep Med. 2013;14(10):943-9.

34. McGrath CL, Kelley ME, Holtzheimer PE, et al. Toward a neuroimaging treatment selection biomarker for major depressive disorder. JAMA Psychiatry. 2013;70(8):821-9.

35. Kennedy SH, Downar J, Evans KR, et al. The Canadian Biomarker Integration Network in Depression (CAN-BIND): advances in response prediction. Curr Pharm Des. 2012;18(36): 5976-89.

36. Ogle NR, Akkerman SR. Guidance for the discontinuation or switching of antidepressant therapies in adults. J Pharm Pract. 2013;26(4):389-96.

37. Berlin I, Lavergne F. Early predictors of two month response with mianserin and selective serotonin reuptake inhibitors and influence of definition of outcome on prediction. Eur Psychiatry. 1998;13(3):138-42.

38. Nierenberg AA, Farabaugh AH, Alpert JE, et al. Timing of onset of antidepressant response with fluoxetine treatment. Am J Psychiatry. 2000;157(9):1423-8.

39. Baldwin DS, Stein DJ, Dolberg OT, et al. How long should a trial of escitalopram treatment be in patients with major depressive disorder, generalised anxiety disorder or social anxiety disorder? An exploration of the randomised controlled trial database. Hum Psychopharmacol. 2009;24(4):269-75.

40. Henkel V, Seemuller F, Obermeier M, et al. Does early improvement triggered by antidepressants predict response/ remission? Analysis of data from a naturalistic study on a large sample of inpatients with major depression. J Affect Disord. 2009;115(3):439-49.

41. van Calker D, Zobel I, Dykierek P, et al. Time course of response to antidepressants: predictive value of early improvement and effect of additional psychotherapy. J Affect Disord. 2009; 114(1-3):243-53.

42. Kuk AY, Li J, Rush AJ. Recursive subsetting to identify patients in the STAR*D: a method to enhance the accuracy of early prediction of treatment outcome and to inform personalized care. J Clin Psychiatry. 2010;71(11):1502-8.

43. Rush AJ, Fava M, Wisniewski SR, et al. Sequenced treatment alternatives to relieve depression $\left(\mathrm{STAR}^{*} \mathrm{D}\right)$ : rationale and design. Control Clin Trials. 2004;25(1):119-42.

44. Kim JM, Kim SY, Stewart R, et al. Improvement within 2 weeks and later treatment outcomes in patients with depressive disorders: the CRESCEND study. J Affect Disord. 2011;129(1-3): 183-90.

45. Uher R, Perroud N, Ng MY, et al. Genome-wide pharmacogenetics of antidepressant response in the GENDEP project. Am J Psychiatry. 2010;167(5):555-64.

46. Bares M, Novak T, Kopecek M, et al. The early improvement of depressive symptoms as a potential predictor of response to antidepressants in depressive patients who failed to respond to previous antidepressant treatments. Analysis of naturalistic data. Eur Psychiatry. 2012;27(7):522-7.

47. Gorwood P, Bayle F, Vaiva G, et al. Is it worth assessing progress as early as week 2 to adapt antidepressive treatment strategy? Results from a study on agomelatine and a global meta-analysis. Eur Psychiatry. 2014;53:54-61. 